\section{THE USE OF QUININE HYDROCHLORIDE SOLUTION AS A DRESSING FOR INFECTED WOUNDDS.}

BY KENNETH TAYLOR, M.A., M.D., PATHOLOGIST, AMERICAN AMBULANCE, NECILLY, PARIS.

IT was found in previous experiments in this laboratory that quinine hydrochloride possessed the following quali. tications which are desirable in a dressing solution for infected wounds: ${ }^{1}$

1. It had high bactericidal properties in vitro, especially marked in the case of the $B$. aërogenes capsulatus, against which it was more than ten times as effective as carbolic acid. It reduced the mortality of experimental gaseous gangrene in guinea-pigs from 100 to 41 per cent. It did not inhibit phagocytosis in the animals treated.

2. It had a strong antiferment (antitryptic) action in vitro, preventing the digestion of proteins and the consequent production of a medium favourable to bacterial growth.

3. It formed no stable chemical combination with proteins, so that its activity was not greatly reduced by the presence of serum or pus.

4. It was practically non-irritating when applied in effective concentration to exposed tissues, or even when injected hypodermically into muscles.

5. It was non-toxic when used in adequate dosage, producing no systemic reaction except a frequently desirable antipyretic effect.

6 . The cost of the materials used was not prohibitive for general application.

7. It presented no difficulties of preparation and did not alter its composition on standing.

On the basis of these observations the treatment of clinical cases of infected wounds has been undertaken.

The majority of the cases have been subjected to wet dressings of a 1 per cent. solution of quinine hydrochloride in cold boiled water. The same solution has been used for instillation. In about 12 cases a continuous drip of ${ }_{10}^{1}$ per cent. solution of quinine hydrochloride, with the addition of 1 per cent. of hydrochloric acid or of 1 per cent. of alcohol, has been used. In addition to these procedures, a few patients have received hypodermic injections into the tissues about the wounds of the same solution as that used for wet dressings.

About 125 cases of infection have so far been treated in this hospital with one of these solutions. These cases have been for the greater part fairly fresh wounds, having usually been received within forty-eight aours of injury. Most of them (70 per cent.) have been heavily infected with the gas bacillus, and all with staphylococci, streptococci, and the usual flora of putrefactive bacteria. About one-half have had open fractures of long bones of arm or leg.

\section{Clinical Course of the Infections.}

Most of the wounds treated with quinine solution have run a very favourable course. There has been a rapid improvement in the appearance of the wound itself, manifested by the speedy disappearance of the sloughs, by the vanishing of the putrefaction odour (frequently within twenty-four or forty-eigbt hours), by a marked decrease in the discharge, and by the unusually rapid appearance of red vascular granulaiions.

Nearly all the most serious wounds showing heavy mixed infections, or those showing the presence of the gas bacillus, have been placed upon this treatment immediately, so that control cases have been difficult to obtain. Ten patients, however, on admission to the hospital wero treated with dressings other than quinine; the treatment was continued for a number of days and then changed to the quinine solution. All of them showed sudden improvement associated with the change. In three instances there was an almost immediate drop in temperature coincident with a marked improvement in the appearance of the wound.

Bacteriological Findings.

An examination of the discharges from the wounds in a series of 35 cases showing the heariest infections has been conducted by Dr. Bertram H. Buxton, of this labora. tory, with a view to ascertaining the time at which the gas bacillus disappeared from the wounds. The annexed table shows the result of his investigations. It may be noted that the incidence of this bacillus in the wounds decreases rapidly under the treatment. All the 7 cases in which the gas bacillus was obtained in the last culture from the wound showed clinical improvement and a great decrease in the number of bacilli present.

Table of Cases Infected by B. aërogenes capsulatus, and Treated by Dressings of Quinine Hydrochloride.

\begin{tabular}{c|c|c|c}
\hline $\begin{array}{c}\text { No. } \\
\text { of } \\
\text { Cases. }\end{array}$ & $\begin{array}{c}\text { Interval between } \\
\text { First and Last } \\
\text { Cultures. }\end{array}$ & $\begin{array}{c}\text { B. ärogenes capsu- } \\
\text { latus Present in } \\
\text { Last Culture. }\end{array}$ & $\begin{array}{c}\text { B. aërogenes capsil- } \\
\text { latus Absent in } \\
\text { Last Culture. }\end{array}$ \\
\hline 2 & 4 days & 1 case & 1 case \\
12 & 10 days & 5 casses & 7 cases \\
15 & 14 days & 1 case & 14 cases \\
6 & 18 days & 0 cases & 6 cases \\
\hline
\end{tabular}

In previous examinations of cases infected with the gas bacillus in this hospital it has been found that the organism has usually persisted for three weeks or more in wounds of the same type-for instance, in a group of 19 cases treated with dressings other than quinino hydrochloride the bacillus was found

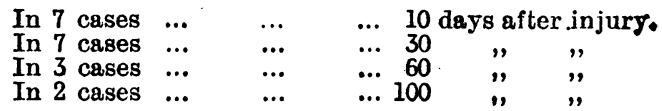

There appears to be associated with the decrease in the incidence of the B. aërogenes capsulatus in the wounds treated with quinine a progressive increase in the occurrence of the $B$. pyocyaneus. It was found in the first series of cultures that while the latter bacillus was reported in only 4 out of 35 cases at the first cultures, it was reported in 14 out of the same 35 cases in the last cultures. This bacillus seemed to occur much earlier in wounds treated with quinine dressings than in those treated with other routine antiseptic solutions, (It was found in previous investigations in this laboratory on the sterilization of pus with quinine that the $B$. pyocyaners was the most resistant to this alkaloid of the organisms commonly present in pus.)

Special attention has been paid to the leucocytes present in the discharges from the quinine-treated wounds. These cells have shown a healthy appearance associated with the clinical improvement of the wounds, and frequently active phagocytosis of the organisms present, with the exception of the $B$. aërogenes capsulatus, which is rarely ingested by them.

As a result of the observations on the cases treated by these solutions and the bacteriological examination of the wounds, considerable confidence has been placed in the use of quinine as an antiseptic as well as in its specific action upon the gas bacillus. Surgeons at this and other hospitals where the treatment is in use have consistently reported that the wounds treated by wet dressings of 1 per cent. quinine hydrochloride have done unusually well. Several have reported that they are using this dressing exclusively for all types of fresh wounds.

\section{Summaky.}

1. The clinical experience with quinine hydrochloride has been consistent with the laboratory work previously reported.

2. It has been active in ridding heavily contaminated wounds of the gas bacillus.

3. The solution has appeared to act as an antiferment inhibiting the activity of the putrefactive organisms and reducing the offensive odour associated with them.

4. The solution has not appeared to be precipitated by the serum or discharge from the wounds in the case of the 1 per cent. solution. At this concentration it had been found previously that its bactericidal activity in vitro was not very markedly diminished by the presence of serum or pus. The is per cent. solution has occasionally shown precipitation when used without the addition of $\frac{1}{10}$ per cent. bydrochloric acid or of 1 per cent. of alcohol. The. addition of neither of these drugs has appreciably increased 
the bactericidal activity of the quinine solution in vitro, but appears to prevent precipitation of the alkaloid caused by the strongly alkaline discharge present in some profusely suppurating wounds.*

5. There has been no report of any injurious effects from the application of any of the methods described above.

6 . There has been reported no unfavourable systemic reaction from the absorption of the solution, while a few cases have shown a desirable decline in temperature associated with the substitution of quinine dressings for others.

The cost of the solution should not prevent its fairly general use. The 1 per cent. solution amounts to about $8 d$. per litre-rather more expensive than some of the other solutions in general use-but as it las seemed con. siderably to shorten the course of convalescence, it is not regarded as an expensive solution. It is further believed, from observations on in vitro experiments, that a solution of $\frac{1}{2}$ per cent. would be effective on the wounds if the acidity of the solution were increased by the addition of ${ }_{2}^{1} v$ per cent. of hydrochloric acid. This latter solution has not been used clinically except on a few cases, each of which has run a satisfactory course.

It is a pleasure to acknowledge the laboratory's financial assistance from Mr. Robert Walton Goelet; the co-operation of Dr. Joseph A. Blake, in whose wards much of the work has been conducted; and the aid of Dr. Bertram H. Buxton, who has made the bacteriological examination of the wounds.

REFREREC.

1 The Use of Quinine in the Treatinent of Experimental Gaseous Gaugrene, Lancet, September 4th, 1915, p. 538 .

\section{NOTES ON TRAUMATIC AND ARTERIO-VENOUS ANEURYSM.}

By Captain F. L. A. GREaves, R.A.M.C.

The following notes of one case of traumatic aneurysm and four of arterio-venous aneurysms will afford an opportunity for discussing some of the symptoms and treatment of this condition.

CASE 1.-Traumatic Aneurysm of Right Poptiteal Artery.

Multiple shrapnel wounds sustained 'July 25 th ; admitted
three days later. Severe compound confminuted fracture of three days later. Severe compound comiminuted fracture of
left tibia and fibula badly infected by 13 . perfringens. T'here left tibia and fibula badly infected by 13 . perfringens. I'here
were smaller wounds at the back of both popliteal spaces, which were much cleaner, and no operation was done on them, but the left leg was carefully cleancd up and wide drainage established. Continuous irrigation with isotonic saline was employed and the leg speedily became healthy.

About three weeks after admission a swelling was noticed in the right popliteal space, lying just internal to the biceps tendon. The skin was hotter over it than normal, the swelling was soft and fluctuated, but there was marked expansile pulsation. No thrill was present, palpation elicited slight pain; movement of flexion of the right knee-joint was limited. A movement of flexion of the right knee-joint was limited. A
definite systolic bruit was heard with the stethoscope over the swelling; this was conducted upwards to the external iliac artery and downwards to the posterior tibial at the ankle and also to the dorsalis pedis. Neither of these vessels was also to the dorsalis pertis. Neither of these vessels was
palpable. The foot was quite warm and no sweling or pain was present in the leg.

For two days the swelling increased slightly and was softer and more superficial; after this it became gradually and progressively harcler, more defined, and more circumscribed. The skin became cooler and the pulses eventually returned in the posterior tibial and dorsalis pedis arteries. At the time of receipt of injury there was no haemorrhage from this right popliteal wound. An $x$-ray plate showed the presence of a bit of metal.

The patient was evacuated for England on September 25th (nine weeks after injury) with a small, firm aneurysm of the right popliteal artery still contracting, $\Omega$ united left tibia and fibula, and all wounds healed.

Treatment of the aneurysm was simple rest, obtained by pillows and sandbags, with the limb in position of slight flexion and elevation.

CASE II.-Arterio-vonous Aneurysm of Third Part of Subclavian Vessels.

Single round from bomb explosion, August 27th, 1915, on the right side of the neck, with severe haemorrhage, which was arrested by a private soldier compressing the wound with his

* It was reported from one hospital that this solution showed the fornation of a precipitate on standing, but it was found that the water used came from a very highly alkaline spring. No difficulty
was experienced after the addition of a fow drops of hydrochloric acid was experie
to the litre. thumb. Admitted next day, very pale and faint, rapid respiration, rapid and feeble pulse; perspiring slightly about forehead and nostrils. There was a wound horizontally placed above th junction of the inner third and outer two-thirds of the right clavicle, and swelling of supraclavicular triangle, and upper pectoral region. Marked pulsation was felt above and below the clavicle and very marked thrill, like the purr of a cat. On auscultation with a stethoc cope a loud continuous bruit was heard like the whirr of nachinery; the point of maximum intensity of thrill and bruit was just below the mid-point of the clavicle. The bruit was well heard all over the right pectoral region and conducted to the axillary and radial arteries. No radial pulse could be detected, and only a possible brachia pulse. The right hand and arm were cold. He had pain in his to a point $2 \frac{1}{3}$ in. above the elbow. Presumably, therefore, a to a point $2 \frac{1}{3}$ in above the elbow. Presumably,
severe injury to the brachial plexus had occurred.

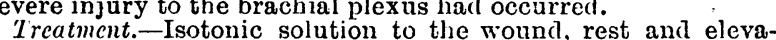
Treatmcnt.-Isotonic solution to the wound, rest and elevation and warmth to hand and arm; he was kept very quiet
in semirecumbent posture. The after-history was that of a in semirecumbent posture. 'The after-history was that of a the pulse-rate dropped very slowly, the pain in hand and arm -diminished in intensity, but never quite disappeared. No porve of motion or improved sensation occurred, and where he picked at the skin of two fingers he developed very indolent little sores. There was never any pulmonary complication. He was evacuated to England on September 5th, 1915, in good general condition with the signs of arterio-venous aneurysm much localized, but with a completely useless right arm. $X$ rays showed a bit of metal lying vertically below the mid-clavicle anterior to the upper part of the scapula, below and internal to the coracoid process.

CASE III.-Arterio-venous Aneurysm of Second Part of Right Subcla "Artery.

Single wound on right siue of neck from piece of shrapnel from a " whizz-bang," sustained on September 5th, 1915. Not much immediate bleeding. When admitted, three days later, he was pale, with rapid pulse and respiration; he had not coughed up any blood; general appearance that of internal haemorrhage. Small entrance wound, moderately septic on the right side of Small entrance wound, moderately septic on the right side of
the neck close to the inner end of the clavicle. There was a the neck close to the inner end of the clavicle. There was a
small amount of swelling and ecchymosis over the clavicle and small amount of swelling and ecchymosig over the clavicle and upper pectoral region, and well marked thrill and bruit very brachial plexus was damaged; he could flex his fingers and use his biceps group. Other muscle groups of arm and hand were paralysed. There was dullness, loss of rocal fremitus, and resonance at the right base (? right haemothorax). The bruit was heard with a stethoscope all over the right chest and conducted to the radial artery at the wrist. The radial pulse was present, but feeble; no swelling of hand or arm.

The general condition improved with treatment similar to that in Case II; circulation in radial pulse also soon improved. The thrill and bruit became more localized to a point internal to the mid.clavicular line. He was evacuated to England on October 6th, 1915. The fluid in the chest had cleared up, and his general condition was excellent; there was no improvement in sensation or muscular power in the right arm. $X$ rays showed a bit of metal posterior to the fifth rib 1 in in. from thio front of the chest.

In neither of these, two cases could the patient hear his own bruit.

Case IV.-Arterio-venous Ancurysm of Axillary Vessels. Snstained a circular wound over the upper part of the right deltoid region from a high explosive shell on September 6th, 1915. Some smart haemorrhage was stopped by dressing. When admitted, two days later, the wound was moderately septic ; there was general swelling of right shoulder and sliglit ecchymosis, and a diffuse swelling extending from the axilla a short distance down the arm on the inner side. Expansile short distance down the arm on the inner side. Expansile
pulsation was seeil and felt. A very marked thrill was present. pulsation was seen and felt. A very marlied thill was present. the sivelling and conducted to the root of the neck and down to the radial at the wrist. The distal circulation was good to the radial at the wrist. The distal circulation was good; tho radial artery was easily felt. There was an area of ulnar distribution. No loss of motion. In addition to the swelling mentioned, a tumour (? foreign body) could be felt high up on the inner wall of the axilla. No pulmonary complications were present.

The swelling gradually contracted and became harder and more'defined ; the point of maximum intensity of thrill and bruit also became more localized, the ulnar anaesthesia gradually passed off, and he was evacuated to England on October 6th. $X$ rays showed a metal fragment on the outer side of the chest wall, high up in the axilla, with eight other tiny fragments lying along the track of the larger piece.

CASE V.-Arterio-venous Aneurysm (Femoral).

Struck by shrapnel bullet on outer side of the left thigh on September 6th, 1915; not much haemorrhage at the time. Admitted two days later with nearly clean entrance wound just in front of the left great trochanter. The general condition was good, but he complained of pain down the front of the left thigh and leg-distribution of long saphenous nerve. A probe introduced into the wound failed to reach the bullet. An $x$-ray photograph showed a shrapnel bullet in the adductor region. An anaesthetic was given and, the dressing being moved, 